\title{
Revisitando definições e naturezas da intersetorialidade: um ensaio teórico
}

\author{
Revisiting definitions and natures of intersectoriality: \\ a theoretical essay
}

Nília Maria de Brito Lima Prado (https://orcid.org/0000-0001-8243-5662) ${ }^{1}$

Rosana Aquino (https://orcid.org/0000-0003-3906-5170) ${ }^{2}$

Zulmira Maria de Araújo Hartz (https://orcid.org/0000-0001-9780-9428) ${ }^{3}$

Hebert Luan Pereira Campos dos Santos (http://orcid.org/0000-0003-2722-7945) ${ }^{4}$

Maria Guadalupe Medina (https://orcid.org/0000-0001-7283-2947) ${ }^{2}$

\footnotetext{
${ }^{1}$ Programa de PósGraduação em Saúde Coletiva, Instituto Multidisciplinar em Saúde, Universidade Federal da Bahia. R. Hormindo Barros 58, Quadra 17, Lote 58, Candeias. 45029-094 Vitória da Conquista BA Brasil. nilia.prado@ufba.br ${ }^{2}$ Programa de PósGraduação em Saúde Coletiva, Instituto de Saúde Coletiva, Universidade Federal da Bahia. Vitória da Conquista BA Brasil. ${ }^{3}$ Instituto de Higiene e Medicina Tropical, Universidade Nova de Lisboa. Lisboa Portugal. ${ }^{4}$ Instituto Multidisciplinar em Saúde, Universidade Federal da Bahia. Vitória da Conquista BA Brasil.
}

\begin{abstract}
This theoretical essay addresses definitions and central characteristics of intersectoral actions for health. It aims, therefore, to reflect on the knowledge produced about the conceptions and nature of health intersectoral actions. An integrative review was conducted for the construction of the analyses from publications referring to the 2000-2019 period identified in the Lilacs, SciELO, Web of Science, and Science Direct databases. The corpus included 12 papers, with aspects related to the aim, purpose or method of intersectoral action under analysis, outlining similarities and contrasts between definitions. Given the opacity in the construction of definitions, we had to point out a socio-historical construction of the concepts, and that the provisional definitions express a certain blurring or erasure in dispute within the field of health that can lead to different viewpoints and are barriers to include the implementation of intersectoral actions related to health promotion in daily practices. Such reflections derived a set of propositions about the nature of intersectoral health actions that aim to contribute to the debate on the theme.
\end{abstract}

Key words Intersectoral action, Intersectoral health action, Intersectoral collaboration, Review
Resumo Este ensaio teórico aborda definições e características centrais das ações intersetoriais para a saúde. Tem como objetivo, portanto, refletir sobre o conhecimento produzido a respeito das concepções e da natureza das ações intersetoriais para a saúde. Para a construção das análises, realizou-se uma revisão integrativa a partir de publicações referentes ao período de 2000 a 2019 identificadas nas bases de dados Lilacs, SciELO, Web of Science e Science Direct. O corpus contemplou 12 artigos, sendo analisados os aspectos relacionados com a finalidade, o propósito ou o método da ação intersetorial, sendo possivvel traçar similaridades e contrastes entre as definições. Diante da opacidade na construção de definições, coube situar que existe uma construção sócio-histórica dos conceitos, mas as definições provisórias expressam certo borramento ou apagamento que se encontram em disputa no interior do campo da saúde e que podem direcionar a distintos pontos de vista e constituir barreiras para incluir a implementação das ações intersetoriais relacionadas à promoção da saúde nas práticas cotidianas. De tais reflexões derivaram um conjunto de proposições acerca das naturezas das ações intersetoriais para a saúde, com vistas a contribuir para o debate sobre a temática.

Palavras-chave Ação intersetorial, Ação intersetorial para a saúde, Colaboração intersetorial, Revisão 


\section{Introdução}

Desde a década de 1970, reforça-se o ideário da intersetorialidade como estratégia para o fortalecimento de ações de promoção no âmbito da atenção primária à saúde (APS), especialmente após a publicação da Declaração de Alma-Ata. Esse documento, fruto da Conferência Internacional sobre Cuidados Primários de Saúde, inclui a pactuação da meta para alcançar níveis de saúde equitativos, conhecida mundialmente como Saúde para Todos ${ }^{1}$.

A abordagem intersetorial da saúde tende a ser associada ou equacionada hoje com a expressão health in all policies (HiAP), traduzida como "saúde em todas as políticas", um termo cunhado no final da década de 1990 como sinônimo de fomentar uma estratégia política e um indicador comum de desenvolvimento. Mas que, é importante salientar, se distingue de outras iniciativas intersetoriais para promover a equidade na saúde, por ser coordenada por estruturas e mecanismos formais de governos e estar explicitamente vinculada a políticas ou agendas governamentais de longo prazo ${ }^{2,3}$.

Ao considerar que a multiplicidade e complexidade das necessidades sociais requer intervenções intersetoriais, essas passam a ser priorizadas como alternativa para propiciar mudanças de cultura organizacional, viabilizando a ampliação do escopo de ações e reorganização dos sistemas locais de saúde, a convergência de interesses entre os profissionais e a construção de alianças na implementação de ações, com a finalidade de responder de maneira mais eficaz e eficiente às demandas da população $0^{4-6}$. Diversos estudos têm identificado o reforço da intersetorialidade como estratégia de gestão em saúde, especialmente em sistemas descentralizados, que favorecem a articulação com outros setores, considerando princípios como território, regionalização e integralidade da atenção $0^{7-10}$.

No entanto, o conhecimento disponível sobre a temática centra-se em aspectos políticos e operacionais para a consecução de uma agenda política universal. Não contemplam os aspectos epistemológicos ou relacionados ao cerne da natureza ou características centrais de uma ação intersetorial em saúde ${ }^{11-15}$. Ecoando algumas críticas, apesar do amplo reconhecimento da importância fundamental da ação intersetorial, permanece a escassez de evidências, uma polissemia conceitual do termo, ausência de categorias operacionais ou estrutura conceitual que permitam a ampliação de abordagens para um envolvimento intersetorial significativo e a implementação e planejamento de políticas públicas transversais específicas em vários níveis de governanç ${ }^{10,15,16}$.

Ante a diversidade de interpretações e definições distintas relacionadas às ações intersetoriais vinculadas à saúde, o presente ensaio teórico objetivou refletir a respeito do conhecimento produzido quanto às concepções e características centrais das ações intersetoriais para a saúde. Para tanto, as questões que balizaram as reflexões foram: como a literatura científica tem definido as ações intersetoriais? Como pode ser caracterizada a natureza de uma ação intersetorial para a saúde?

Apesar de a ação intersetorial constituir um tema recorrente na saúde pública, não existe uma teoria explícita para determinar que tipos de ações intersetoriais são viáveis sob diferentes cenários, ou o tipo de ação intersetorial necessário para abordar os determinantes sociais da saúde e reduzir as iniquidades em saúde ${ }^{16}$. Com isso, reforça-se a imprescindibilidade da adoção de uma abordagem com base em premissas conceituais padronizadas, e que possam fortalecer o planejamento de ações derivadas de parcerias com a sociedade civil, suscitar políticas transparentes, multissetoriais e orientadas para a equidade em saúde.

\section{Metodologia}

Este trabalho tem cunho qualitativo, trata-se de um ensaio teórico reflexivo pautado na construção de uma análise crítica da literatura internacional e nacional sobre definições e natureza das ações intersetoriais direcionadas à saúde.

Os elementos de análise foram extraídos de uma revisão integrativa da literatura que permitiu identificar, a partir das experiências descritas em artigos e documentos técnicos, as características específicas e o escopo das ações intersetoriais em saúde.

Tomamos como orientação para a ampliação das referências a exploração de documentos técnicos e artigos nacionais e internacionais nas seguintes bases de dados bibliográficas: SciELO, Scientific Electronic Library Online - Lilacs, Web of Science e Science Direct, utilizando os unitermos previamente identificados no descritores em ciências da saúde (DeCS) e Medical Subject Heading (MeSH), sendo eles: health promotion, intersectoral action, intersectoriality, health in all policies, e seus correlatos em português, inglês e espanhol, assim como os termos indicados como sinônimos. 
Em seguida, fez-se a elaboração das sintaxes de busca mais adequadas à localização dos documentos nas bases de dados selecionadas, a saber: (health promotion) AND (Ação Intersetorial OR intersectoral action for health) AND (Primary Health Care AND Intersectoral Action) AND (Intersectoral action OR Intersect ${ }^{*} \mathrm{OR}$ intersectoriality $\mathrm{OR}$ intersectoral coordination $\mathrm{OR}$ intersectoral cooperation) AND (Interserct ${ }^{\star}$ OR Intersectoral action OR Intersectoral) AND (intersectoral action OR Intersectoral ) AND (Health OR Saúde) AND (Health Primary Care OR Atenção Básica) AND (Promoção da Saúde AND Coordenação Intersetorial) AND (Health Promotion AND Intersectoral Action) AND (Atención Primaria de La Salud) AND (Promoción de La Salud) AND (Acción Intersectorial). Os dados foram coletados no período de junho de 2019 a dezembro de 2019.

Uma base de dados única foi delineada em uma planilha do Microsoft Excel que reuniu um total de 1.029 documentos identificados. Esses foram compilados e submetidos a um processo de seleção, considerando-se os seguintes critérios de inclusão: (1) pertinência ao tema; (2) textos completos publicados entre 1978 (publicação da Declaração de Alma-Ata/Conferência Internacional de Cuidados Primários à Saúde) e dezembro de 2019; (3) ter seu resumo disponível nas bases de dados incluídas neste estudo; e (4) ser estudo empírico e/ou de revisão e/ou conceitual na área de promoção da saúde.

Dos 1.029 estudos, 398 foram excluídos por duplicação, restando 631 para leitura de títulos e resumos. Desses, foram excluídos 430 após a leitura de títulos e resumos, restando 201 para leitura dos textos na integra. Após a leitura, foram excluídos 189 publicações que não discutiam os assuntos considerados centrais nesta revisão, resultando em 12 artigos para o desenvolvimento final da revisão.

Os 12 artigos selecionados foram sistematizados conforme o ano de publicação, país do estudo, local, tipo de estudo, delineamento da pesquisa, descrição de um conceito e de características de uma ação intersetorial para a saúde. Após a análise dos artigos, dois eixos temáticos foram criados como categorias emergentes: a) premissas explicitas nas sinonímias da ação intersetorial para a saúde; b) finalidade, propósito ou método explícitos nas definições de ação intersetorial; e c) a natureza das ações intersetoriais.

Entre os estudos selecionados, pôde-se observar que os objetivos esperados vão desde a integração administrativa, formal e sistemática, por meio da integração de objetivos e processos administrativos e a partilha de recursos, responsabilidades e ações, até o objetivo de desenvolvimento conjunto de políticas de saúde, sociais e educacionais, buscando alcançar uma forma de integração com alto grau horizontalidade nos sistemas e serviços de saúde (Quadro 1).

\section{Premissas explícitas nas sinonímias para o termo ação intersetorial para a saúde}

Com relação ao conjunto de sinonímias utilizadas como forma de expressão da ação intersetorial, parece, na verdade, expor diferentes níveis de gestão intersetorial ou diferentes intensidades de relações interinstitucionais. Os resultados demonstraram o emprego de sinônimos do termo ação intersetorial $^{17-25}$; intersetorialidade ${ }^{26-28}$; ação intersetorial para a saúde ${ }^{29}$. E de sinonímias, a saber: cooperação intersetorial ${ }^{28,30-33}$; coordenação intersetorial 20,28,33; colaboração intersetorial ${ }^{32,34-36}$; colaboração interorganizacional $^{32}$ e integração intersetorial ${ }^{33}$, evidenciando uma polissemia conceitual.

A análise dos artigos selecionados permitiu assinalar duas premissas acerca da noção de ação intersetorial. A primeira é de caráter político e propõe que a integração de diversos setores permita a busca de soluções mais integrais - ideia de soluções globais de desenvolvimento ${ }^{20,23-25,37,38}$. A segunda é de natureza técnica, uma vez que acredita que a integração entre os setores permite que as diferenças entre eles possam ser usadas produtivamente para resolver problemas sociais ${ }^{19,20,26-28}$.

No percurso desse debate, torna-se crucial identificar se o propósito de união ou esforço entre diferentes setores para estabelecer uma cooperação direcionada a uma questão ou problema comum prevaleceu entre as definições identificadas neste estudo ${ }^{19,24,25,26,32,34-36}$.

As definições que propuseram integração setorial para viabilizar o desenvolvimento de ações globais tinham como ponto de convergência a mobilização de esforços políticos e o intuito de redução de custos e racionalização de ações entre os diferentes setores governamentais, visando a sinergia de competências e estratégias para alcançar resultados mais abrangentes ${ }^{18,25,26,33}$, que não seriam possíveis de forma setorial ${ }^{26,32,34,36,38,39}$.

Contudo, os resultados de uma revisão de escopo realizada por Shankardass e colaboradores $^{32}$ propiciou a proposição de uma escala para mensuração do nível de articulação intersetorial, considerando o nível 0 (mais frágil): compartilhamento de dados e informações muitas vezes unidirecional; nível 1: cooperação - para otimizar recursos; nível 2: coordenação com maior ho- 
Quadro 1. Descrição dos estudos incluídos, segundo autor, ano de publicação, país de localização do primeiro autor, título, tipo de estudo e conceito de ação intersetorial abordado na publicação.

\begin{tabular}{|c|c|c|c|}
\hline $\begin{array}{l}\text { Autor, ano, país } \\
\text { de origem }\end{array}$ & Título & $\begin{array}{l}\text { Tipo de } \\
\text { estudo }\end{array}$ & Definição/termo abordado \\
\hline $\begin{array}{l}\text { Mannheime et al. } \\
\text { (2007), Suécia }\end{array}$ & $\begin{array}{l}\text { Introducing } \\
\text { Health Impact } \\
\text { Assessment: an } \\
\text { analysis of political } \\
\text { and administrative } \\
\text { intersectoral working } \\
\text { methods }\end{array}$ & Empírico & $\begin{array}{l}\text { "Intersectoral Action for Health (IAH), which is } \\
\text { defined as 'action in which the health sector and } \\
\text { other relevant sectors of the economy collaborate } \\
\text { or interact to pursue health goals" (Citing WHO } \\
\text { 1998)/ação intersetorial para a saúde }\end{array}$ \\
\hline $\begin{array}{l}\text { Lal, Mercier } \\
\text { (2009), Canadá }\end{array}$ & $\begin{array}{l}\text { Intersectoral action } \\
\text { to employ individual } \\
\text { with mental illness: } \\
\text { Lessons learned from } \\
\text { a local development } \\
\text { initiative }\end{array}$ & Empírico & $\begin{array}{l}\text { "Collaboration between different sectors of the } \\
\text { community"/colaboração intersetorial }\end{array}$ \\
\hline $\begin{array}{l}\text { Zunzunegui, } \\
\text { Béland } \\
\text { (2010), Canadá }\end{array}$ & \begin{tabular}{|l|} 
Politiques \\
interstoriales/ \\
Informe SESPAS \\
2010 \\
\end{tabular} & Empírico & $\begin{array}{l}\text { 'Unir esfuerzos para conseguir mejores resultados } \\
\text { que los que se obtendrían trabajando de forma } \\
\text { aislada"/ação intersetorial }\end{array}$ \\
\hline $\begin{array}{l}\text { Shankardass et al. } \\
\text { (2012), Canadá }\end{array}$ & $\begin{array}{l}\text { A scoping review } \\
\text { of intersectoral } \\
\text { action for health } \\
\text { equity involving } \\
\text { governments }\end{array}$ & Teórico & $\begin{array}{l}\text { "Cooperation Interaction between sectors to achieve } \\
\text { greater efficiency in their actions. Aims to optimize } \\
\text { resources while establishing formalities in the work } \\
\text { relationship; results in some loss of autonomy for } \\
\text { each sector/cooperação intersetorial; } \\
\text { "Coordination Adjusting the policies and programs } \\
\text { of each sector to improve efficiency and effectiveness } \\
\text { leads to increased horizontal networking among } \\
\text { sectors. Usually uses a shared financing source which } \\
\text { creates synergies within administration but leads } \\
\text { to a greater dependence between sectors and loss of } \\
\text { autonomy"/coordenação intersetorial; } \\
\text { "Integration is a political process where a new } \\
\text { policy or program (representing the work of } \\
\text { multiple sectors) is defined in conjunction with } \\
\text { other sectors. This entails the integration of } \\
\text { objectives and administrative processes and the } \\
\text { sharing of resources, responsibilities and actions. } \\
\text { This ultimately results in the collapsing of "closed } \\
\text { fiefdoms"/integração intersetorial }\end{array}$ \\
\hline $\begin{array}{l}\text { Clavier et al. } \\
\text { (2013), Canadá }\end{array}$ & $\begin{array}{l}\text { L'action } \\
\text { intersectorielle } \\
\text { en santé publique } \\
\text { ou lorsque les } \\
\text { institutions, les } \\
\text { intérêts et les idées } \\
\text { entrent en jeu }\end{array}$ & Teórico & $\begin{array}{l}\text { "Le concept d'intersectorialité est défini ici au sens } \\
\text { d'une action conjointe entre des acteurs relevant } \\
\text { de deux ou plusieurs secteurs d'action publique } \\
\text { (citando Muller, 2004). Nous utiliserons l'expression } \\
\text { d'action intersectorielle pour signifier les pratiques } \\
\text { d'intersectorialité sur le terrain"/intersetorialidade }\end{array}$ \\
\hline $\begin{array}{l}\text { Buse (2013), } \\
\text { Canadá }\end{array}$ & $\begin{array}{l}\text { Intersectoral action } \\
\text { for health equity as } \\
\text { it relates to climate } \\
\text { change in Canada: } \\
\text { contributions from } \\
\text { critical systems } \\
\text { heuristics }\end{array}$ & Teórico & $\begin{array}{l}\text { A recognised relationship between part or parts } \\
\text { of the health sector with part or parts of another } \\
\text { sector which has been formed to take action on an } \\
\text { issue to achieve health outcomes (or intermediate } \\
\text { health outcomes) in a way that is more effective, } \\
\text { efficient or sustainable than could be achieved by the } \\
\text { health sector acting alone (citing WHO, 1997)/ação } \\
\text { intersetorial }\end{array}$ \\
\hline
\end{tabular}


Quadro 1. Descrição dos estudos incluídos, segundo autor, ano de publicação, país de localização do primeiro autor, título, tipo de estudo e conceito de ação intersetorial abordado na publicação.

\begin{tabular}{|c|c|c|c|}
\hline $\begin{array}{c}\text { Autor, ano, país } \\
\text { de origem }\end{array}$ & Título & $\begin{array}{l}\text { Tipo de } \\
\text { estudo }\end{array}$ & Definição/termo abordado \\
\hline $\begin{array}{l}\text { Freiler et al. } \\
\text { (2013), Canadá }\end{array}$ & $\begin{array}{l}\text { Glossary for the } \\
\text { implementation of } \\
\text { Health in All Policies } \\
\text { (HiAP) }\end{array}$ & Teórico & $\begin{array}{l}\text { "A recognised relationship between part or parts } \\
\text { of the health sector with part or parts of another } \\
\text { sector which has been formed to take action on an } \\
\text { issue to achieve health outcomes (or intermediate } \\
\text { health outcomes) in a way that is more effective, } \\
\text { efficient or sustainable than could be achieved by } \\
\text { the health sector acting alone (Citing WHO, 1997)/ } \\
\text { ação intersetorial; } \\
\text { "Is the coordination of various sectors towards the } \\
\text { improvement of health equity, HiAP should be } \\
\text { considered the most administratively integrated, } \\
\text { formal and systemically-focused form of } \\
\text { intersectoral action. Intersectoral action may also be } \\
\text { referred to as intersectoral initiatives, intersectoral } \\
\text { approach or whole-of-government approach } \\
\text { (citing Public Health Agency of Canada, 2009)"/ } \\
\text { coordenação intersetorial }\end{array}$ \\
\hline $\begin{array}{l}\text { Anaf et al. (2014), } \\
\text { Austrália }\end{array}$ & $\begin{array}{l}\text { Factors shaping } \\
\text { intersectoral action } \\
\text { in primary health } \\
\text { care services }\end{array}$ & Empírico & $\begin{array}{l}\text { "A recognized relationship between part or parts of } \\
\text { the health sector with part or parts of another sector } \\
\text { which has been formed to take action on an issue } \\
\text { to achieve health outcomes (or intermediate health } \\
\text { outcomes) in a way that is more effective, efficient } \\
\text { or sustainable than could be achieved by the health } \\
\text { sector acting alone"/ação intersetorial }\end{array}$ \\
\hline $\begin{array}{l}\text { Weiss, Lillefjell, } \\
\text { Magnus } \\
\text { (2016), Noruega }\end{array}$ & $\begin{array}{l}\text { Facilitators for the } \\
\text { development and } \\
\text { implementation of } \\
\text { health promoting } \\
\text { policy and programs - } \\
\text { a scoping review at the } \\
\text { local community level }\end{array}$ & Empírico & $\begin{array}{l}\text { "Working with more than one sector of society } \\
\text { to take action on an area of shared interest. } \\
\text { Sectors may include government departments } \\
\text { such as health, education, environment and } \\
\text { justice; ordinary citizens; non-profit societies or } \\
\text { organizations; and business"/ação intersetorial }\end{array}$ \\
\hline $\begin{array}{l}\text { Wimmer, } \\
\text { Figueiredo (2006), } \\
\text { Brasil }\end{array}$ & $\begin{array}{l}\text { Ação coletiva } \\
\text { para qualidade de } \\
\text { vida: autonomia, } \\
\text { transdisciplinaridade } \\
\text { e intersetorialidade } \\
\end{array}$ & Empírico & $\begin{array}{l}\text { "A intersetorialidade é uma prática integradora de } \\
\text { ações de diferentes setores que se complementam e } \\
\text { interagem, para uma abordagem mais complexa dos } \\
\text { problemas/Intersetorialidade" }\end{array}$ \\
\hline $\begin{array}{l}\text { Dias et al. } \\
(2014) \text {, Brasil }\end{array}$ & $\begin{array}{l}\text { Intersetorialidade e } \\
\text { Estratégia Saúde da } \\
\text { Família: } \\
\text { tudo ou quase nada } \\
\text { a ver? }\end{array}$ & Empírico & $\begin{array}{l}\text { "A intersetorialidade implica em reciprocidades dos } \\
\text { atores que atuam no contexto da saúde coletiva, } \\
\text { o que sugere a ideia de compartilhamentos de: } \\
\text { significados, conhecimentos, compromissos, } \\
\text { valores, afetos, responsabilidades e ações"/ } \\
\text { intersetorialidade }\end{array}$ \\
\hline $\begin{array}{l}\text { Azevedo et al. } \\
\text { (2012), Brasil }\end{array}$ & $\begin{array}{l}\text { Práticas intersetoriais } \\
\text { nas políticas públicas } \\
\text { de promoção de } \\
\text { saúde }\end{array}$ & Teórico & $\begin{array}{l}\text { "Consiste na obtenção de certa unidade, apesar das } \\
\text { diferentes áreas de atuação dos setores envolvidos, } \\
\text { tentando estabelecer vínculos intencionais que } \\
\text { superem a fragmentação e a especialização"/ação } \\
\text { intersetorial }\end{array}$ \\
\hline
\end{tabular}

Fonte: Autores.

rizontalidade e compartilhamento de financiamento; nível 3 (maior intensidade): integração de um processo político em que uma nova polí- tica ou programa apresenta a definição de objetivos, processos administrativos e financiamento compartilhados. 
Ao que parece, as sinonímias compactuam o objetivo de que para implementar e adequar políticas e programas intersetoriais é necessária uma maior articulação horizontal entre os setores, minimizando inconsistências entre eles ${ }^{33}$. Nesse sentido, a articulação intersetorial reflete a proposição de realização de intervenções estruturais mais profundas, que exigem esforços dos setores envolvidos, uma maior integração entre os atores e devem ser orientadas pelas necessidades em saúde, e não por objetivos setoriais ${ }^{32,34-36,40-42}$.

No entanto, em alguns contextos locais, tais fundamentos não conseguem estabelecer relações intersetoriais duradouras ${ }^{34,36,40-44}$ por constituírem apenas uma aproximação momentânea de setores, sem haver uma preocupação, de forma abrangente e definida, quanto à necessidade de mudanças organizacionais, administrativas e operacionais para viabilizar a implementação das ações integradas ${ }^{16,45}$.

Percebe-se que, a natureza da intersetorialidade é definida a partir de três categorias centrais: o nível de inclusão no ciclo de formulação de políticas; o nível de colaboração na implementação de ações; e o nível de mudança nas formas organizacionais preexistentes. A depender do grau de amplitude das ações, se o foco da participação social for apenas a informação ou a colaboração da comunidade com ações específicas de saúde lideradas pelo setor de saúde, tem-se intervenções voltadas para doenças específicas. Por outro lado, quando o padrão predominante de relacionamento com outros setores é a cooperação ou coordenação, o foco das ações intersetoriais passa a ser a prevenção e promoção da saúde. E, por fim, o modelo mais desejável, quando existe um predomínio de relações com outros setores, com base na integração, produz-se intervenções direcionadas a necessidades em saúde alinhadas aos Determinantes Sociais e da Saúde, incluindo os esforços para combater e reduzir as iniquidades em saúde ${ }^{16}$.

\section{Finalidade, propósito ou método explícitos nas definições de ação intersetorial}

A análise empreendida permitiu também apontar a definição da ação intersetorial como finalidade, propósito ou método. A finalidade e o propósito associaram-se ao alcance de resultados cooperativos comuns entre os setores, ao estabelecimento de planejamento compartilhado, à integração de atores e setores chave e ao entendimento mútuo sobre a necessidade de priorização de uma questão específica ${ }^{8,17-20,22,23,26,28,29,32-36,38}$.
Nesse sentido, Shankardass e colaboradores ${ }^{32}$ sistematizaram critérios para definir o escopo das ações intersetoriais para a saúde que contemplam: (a) ação envolvendo colaboração entre mais de um setor governamental; (b) melhoria da equidade como resultado alvo da ação intersetorial, implícita ou explicitamente; (c) intervenção para prevenir as desigualdades na saúde antes que se tornem clinicamente identificável não apenas aumentando o acesso ao cuidado à saúde, mas como um ponto de entrada da ação intersetorial.

Assim, a ação intersetorial objetiva a integração de mecanismos de gestão desenvolvidos em territórios definidos, com capacidade financeira e técnica para articular diferentes setores que se complementam e reúnem atores sociais, saberes, poderes e vontades diversos em uma nova maneira de planejar, executar e controlar a prestação de serviços por meio da confluência para enfrentar as desigualdades sociais e de saúde $e^{23,26-28}$.

\section{As naturezas das ações intersetoriais em saúde}

As definições relacionadas pelas produções analisadas também possibilitou elencar um conjunto de proposições relacionadas aos fatores constitutivos do que se tem denominado ação intersetorial (Quadro 2), que podem ser relacionadas com: 1) o nível de inclusão no ciclo de formulação de políticas - pressupõe o planejamento de ação sobre iniquidade em saúde; 2) o nível de colaboração na implementação de ações - pressupõe agentes sociais com relativa autonomia; agentes com capacidades técnicas especificas; e relações sociais permeadas pelo diálogo; 3) o nível de mudança nas formas organizacionais preexistentes - pressupõe mudanças no processo de trabalho; implementação de ação em um recorte espacial definido; e a possibilidade de replicação em outros espaços sociais.

A abordagem intersetorial geralmente está associada à necessidade de implementação de políticas sociais, de saúde ou educação de âmbito nacional que incorporam em suas diretrizes o propósito de atender a direitos ou questões sociais para uma população ou grupo populacional. A formulação de políticas multissetoriais com base em uma perspectiva integrada pode ampliar os esforços para reduzir a disparidade na saúde muito além da esfera da saúde. Assim, a inclusão da intersetorialidade no ciclo de formulação de uma política contempla ações direcionadas a reduzir iniquidades em saúde. 
Quadro 2. Síntese de proposições e categorias centrais que podem caracterizar a natureza das ações intersetoriais, segundo os artigos selecionados no estudo.

\begin{tabular}{|c|c|c|c|}
\hline & Proposições & Categorias centrais & Referências \\
\hline & $\begin{array}{l}\text { A natureza da ação intersetorial antevê } \\
\text { a redução de iniquidades sociais em } \\
\text { saúde }\end{array}$ & $\begin{array}{l}\text { Nível de formulação de } \\
\text { políticas sobre iniquidade } \\
\text { em saúde }\end{array}$ & $\begin{array}{l}\text { Clavier et al., 2013; Rantala et al., } \\
2014\end{array}$ \\
\hline & $\begin{array}{l}\text { A natureza de uma ação intersetorial } \\
\text { pressupõe o reconhecimento de um } \\
\text { objeto um problema ou um tema } \\
\text { específico }\end{array}$ & & $\begin{array}{l}\text { El Ansari, 2005; Clavier et } \\
\text { al., 2013; Rantala et al., 2014; } \\
\text { Kalegaongar, Brown, 2000; } \\
\text { Sposati, 2006; Axelsson, Axelsson, } \\
\text { 2006; Zunzunegui, Béland, } 2010\end{array}$ \\
\hline & $\begin{array}{l}\text { A natureza da ação intersetorial não diz } \\
\text { respeito ao conteúdo prático desta ação, } \\
\text { mas sim, ao processo de trabalho que } \\
\text { envolve a implementação desta ação }\end{array}$ & $\begin{array}{l}\text { Nível de colaboração dos } \\
\text { atores sociais com relativa } \\
\text { autonomia; agentes com } \\
\text { capacidades técnicas } \\
\text { especificas; e, relações } \\
\text { sociais permeadas pelo } \\
\text { diálogo }\end{array}$ & $\begin{array}{l}\text { Perera, 2006; Shankardass et } \\
\text { al., 2012; Stewart et al., 2006; } \\
\text { Mannheime et al., 2007; } \\
\text { Zunzunegui, Béland, 2010; Freiler } \\
\text { et al., 2013; Rantala et al., 2014; } \\
\text { Tubbing et al., 2015; Rissel, } \\
\text { Rowling, 2000; Keon, Pépin, } 2008 \\
\end{array}$ \\
\hline 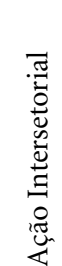 & $\begin{array}{l}\text { A natureza da ação intersetorial implica } \\
\text { em um processo de constituição de } \\
\text { agentes sociais com relativa autonomia } \\
\text { intersubjetiva para a construção } \\
\text { de novas dinâmicas sociais, que } \\
\text { direcionem estratégias em prol da } \\
\text { construção social de um problema }\end{array}$ & & $\begin{array}{l}\text { Mannheimer et al., 2007; } \\
\text { Kalegaonkar, Brown, 2000; Rissel, } \\
\text { Rowling, 2000; Perera, 2006; } \\
\text { Keon, Pépin, 2008; Shankardass } \\
\text { et al., } 2012\end{array}$ \\
\hline 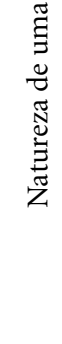 & $\begin{array}{l}\text { A natureza de uma ação intersetorial } \\
\text { preconiza que as relações sociais } \\
\text { sejam permeadas pelo diálogo para } \\
\text { minimizar as contradições simbólicas, } \\
\text { institucionais, profissionais de } \\
\text { diferentes espaços sociais, e possam } \\
\text { viabilizar o estabelecimento de um } \\
\text { equilíbrio em um novo espaço de } \\
\text { decisão }\end{array}$ & & $\begin{array}{l}\text { Kalegaonkar, Brown, 2000; } \\
\text { Perera, 2006; Rissel, Rowling, } \\
\text { 2000; Zunzunegui, Béland, 2010; } \\
\text { Shankardass et al., } 2012\end{array}$ \\
\hline & $\begin{array}{l}\text { A natureza da ação intersetorial } \\
\text { pressupõe a participação de } \\
\text { atores sociais portadores de uma } \\
\text { representação formal }\end{array}$ & & $\begin{array}{l}\text { Rissel, Rowling, 2000; Perera, } \\
\text { 2006; Keon, Pépin, 2008; Stewart } \\
\text { et al, 2006; Shankardass et al., } \\
\text { 2012; Freiler et al., } 2013\end{array}$ \\
\hline & $\begin{array}{l}\text { A natureza de uma ação intersetorial } \\
\text { demanda a integração de agentes com } \\
\text { capacidades técnicas específicas, mas } \\
\text { que se interessam e se mobilizam para } \\
\text { construir um problema em um novo } \\
\text { espaço social }\end{array}$ & $\begin{array}{l}\text { Nível de mudança nas } \\
\text { formas organizacionais } \\
\text { preexistentes }\end{array}$ & $\begin{array}{l}\text { Freiler et al., 2013; Buse et al., } \\
\text { 2013; Tubbing et al., 2014; El } \\
\text { Ansari, 2005; Perera, 2006; } \\
\text { Shankardass et al., 2012; Rissel, } \\
\text { Rowling, 2000; Axelsson, } \\
\text { Axelsson, 2006; Lal, Mercier, 2009; } \\
\text { Kalegaonkar, Brown, } 2000\end{array}$ \\
\hline & $\begin{array}{l}\text { A natureza da ação intersetorial } \\
\text { pressupõe a articulação de agentes com } \\
\text { capacidades técnicas específicas }\end{array}$ & & $\begin{array}{l}\text { Mannheimer et al., 2007; } \\
\text { Kalegaonkar, Brown, 2000; Rissel, } \\
\text { Rowling, 2000; Perera, 2006; } \\
\text { Shankardass et al., } 2012\end{array}$ \\
\hline
\end{tabular}

Fonte: Autores.

Para facilitar a colaboração entre setores, é importante induzir mudanças burocráticas. Inicialmente, pressupõe a organização de estruturas comuns de governança, que incluem o desenvolvimento de parcerias com outros setores e um acordo de colaboração inicial ${ }^{24,33,35}$. Para viabi- 
lizar a articulação intersetorial, em seguida são necessárias a criação de instrumentos de planejamento que viabilizem o trabalho interinstitucional, a definição de responsabilidades e sistemas de informação padronizados para organização e compartilhamento de informações ${ }^{26}$, acompanhamento e avaliação da execução das ações.

Diversos estudos empíricos destacaram a importância de estabelecimento prévio de acordos, sejam eles relacionados à formalização de fluxos de negociação e comunicação $0^{8,17-20,29,34,38}$ ou ao compartilhamento de fontes de financiamento ${ }^{35}$ (orçamento conjunto, cofinanciamento, acordos de transferência de recursos de uma autoridade central, ou redirecionamento de alocações orçamentárias preexistentes, entre outras opções), com a especificação de objetivos e metas ajustadas a um calendário realista. Pressupõem ainda que a tomada de decisão ocorra em espaços de gestão compartilhados, como comitês ou grupos intersetoriais ${ }^{18,19,33,34}$, em que os setores envolvidos na implementação de uma ação intersetorial podem pelo menos expressar seus interesses e tentar conciliar suas diferenças.

Além disso, alguns autores ${ }^{19,27,32}$ ponderam que o desenvolvimento de políticas integradas está relacionado à convergência de esforços entre a saúde e outros setores do governo e a participação da sociedade civil local para direcionar ações para determinada situação prioritária, seja ela um objeto, um tema ou uma necessidade. Dessa forma, as estratégias intersetoriais devem incluir a participação da sociedade civil na execução de ações direcionadas à redução das iniquidades sociais que afetam os níveis de saúde da população.

Nesse sentido, o nível de colaboração na implementação de ações pressupõe um processo de trabalho compartilhado por atores sociais, com relativa autonomia, para representar formalmente as ideias e os interesses dos distintos setores que estão vinculados. Além disso, devem desenvolver habilidades e competências ${ }^{33,35}$ para viabilizar a proposição de estratégias de colaboração $9,18,26,29,33,34,39$ e para desenvolver um processo de trabalho baseado no consenso, no diálogo e na confiança com a nova equipe $e^{26,29,33,38}$.

A intersetorialidade depende da articulação entre agentes de setores diversos, com diferentes saberes e poderes, com vista a enfrentar problemas complexos e produzir efeitos mais significativos na saúde da população. Assim, a ação intersetorial não pode ser espontânea, trata-se de uma ação deliberada, que requer respeito à diver- sidade e às particularidades setoriais, mas pressupõe um processo de trabalho sustentado em um objetivo comum, articulando diferentes níveis de complexidade e de recursos, mobilizando distintos atores sociais para a realização dessa prática.

Dessa maneira, a priorização de questões compartilhadas requer mudanças nas formas organizacionais preexistentes, por meio de treinamento de todos os atores sobre questões relacionadas à saúde pública, incluindo os determinantes sociais da mesma, especialmente quando não há funções intersetoriais ou colaborações prévias no nível local ${ }^{26,35,38}$. Nesse sentido, constitui a articulação de saberes e experiências no planejamento, na realização e na avaliação de políticas, programas e projetos dirigidos a comunidades e a grupos populacionais específicos num dado espaço geográfico com o objetivo de atender às suas necessidades de modo sinérgico e integral.

Contudo, a identificação de necessidades contextualizadas não se refere apenas à demarcação de um território para a operacionalização de ações intersetoriais, antevê também o reconhecimento de aspectos culturais, sociais, econômicos e outros que podem facilitar a proposição e implementação de ações articuladas ${ }^{26,38}$. Assim, deve incluir mudanças nas práticas de trabalho dos agentes envolvidos na gestão e no planejamento a partir de dados de estatísticas sanitárias, demográficas e programáticas relacionadas com a execução da ação intersetorial ${ }^{19,20,26,33}$, bem como a avaliação do alcance dos resultados ${ }^{27}$. Entretanto, para a execução de projetos intersetoriais, é fundamental capacitação técnica dos gestores para criar uma cultura cooperativa nas relações gerenciais e administrativas, aliada à formação técnica para a gestão intersetorial.

Com base nas análises dos estudos incluídos nesta revisão, notou-se que os mesmos não assumiram claramente uma definição, e quando o fizeram, apresentaram interpretações diversificadas. Dessa forma, propõe-se, a partir das reflexões concebidas por este ensaio, uma definição para a ação intersetorial em saúde: Uma ação intersetorial é aquela com capacidade de integrar agentes e distintos setores com capacidades técnicas específicas, que se complementam e, ainda que existam assimetrias de poder, se articulam em espaços de gestão compartilhada, de decisão para o planejamento, execução e avaliação de questões ou necessidades específicas, identificadas com a participação social e direcionadas para a redução de iniquidades sociais em saúde. 


\section{Considerações finais}

Esse ensaio teórico possibilitou ampliar o debate acerca das definições provisórias e as características centrais que modulam a natureza de uma ação intersetorial. A discussão conduzida permitiu apontar fatores inerentes para a implementação das ações intersetoriais em prol da equidade em saúde a partir de experiências que demonstraram a concretização desse potencial em diversos contextos. Assim como subsidiou a construção de um conceito para a ação intersetorial em saúde, que pode constituir uma contribuição te- órica para auxiliar os atores sociais a ultrapassar as contradições inerentes e permitir a ampliação de intenções e práticas direcionadas à implementação de políticas horizontais integradas.

Sem reflexão não há como dar conta do desafio de traduzir o conhecimento científico e compreender os borramentos conceituais, objetivos, métodos e finalidades das ações intersetoriais. Dessa forma, espera-se que esta produção possa proporcionar um novo olhar e novos caminhos para a proposição e implementação de políticas públicas intersetoriais direcionadas a enfrentar as iniquidades em saúde.

\section{Colaboradores}

Todos os autores contribuíram igualmente para a concepção, projeto, aquisição, análise e interpretação dos dados, bem como para a revisão crítica para a aprovação da versão final a ser publicada. Eles são responsáveis por relatar todos os aspectos do trabalho, assegurando que as questões relacionadas à precisão ou à integridade de qualquer parte sejam adequadamente investigadas e resolvidas.

\section{Referências}

1. Brasil. Ministério da Saúde (MS), Secretaria de Políticas de Saúde, Projeto Promoção da Saúde. As cartas da promoção da saúde. Brasília: MS; 2001.

2. Corbin JH. Health promotion, partnership and intersectoral action. Health Promot Int 2017; 32(6):923-929.

3. Ndumbe-Eyoh S, Moffatt $\mathrm{H}$. Intersectoral action for health equity: a rapid systematic review. BMC Public Health 2013;13:1056.

4. Potvin L. Intersectoral action for health: more research is needed! Int J Public Health 2012; 57(1):5-6.

5. Cohn A. Intersectoral governance for health in all policies - structures, actions and experiences. Revista de Direito Sanitário 2013; 14(1):264-267.

6. Akerman M, Sá RF, Moyses S, Rezende R, Rocha D. Intersetorialidade? IntersetorialidadeS! Cienc Saude Colet 2014; 19(11):4291-4300.

7. McCalman J, Bainbridge R, Brown C, Tsey K, Clarke A. The Aboriginal Australian Family Wellbeing Program: a historical analysis of the conditions that enabled its spread. Front Public Health 2018; 6:26.

8. Tubbing L, Harting J, Stronks K. Unravelling the concept of integrated public health policy: Concept mapping with Dutch experts from science, policy, and practice. Health Policy 2015; 119(6):749-759.

9. Chircop A, Bassett R, Taylor E. Evidence on how to practice intersectoral collaboration for health equity: a scoping review. Critical Public Health 2014; 25(2):178-191

10. Dubois A, St-Pierre L, Veras M. Uma revisão do escopo das definições e estruturas de ação intersetorial. Cien Saude Colet; 20(10):2933-2942.

11. Bennett $\mathrm{S}$, Jessani N, Glandon D et al. Compreendendo as implicações dos Objetivos de Desenvolvimento Sustentável para a pesquisa de políticas e sistemas de saúde: resultados de um exercício de definição de prioridades de pesquisa. Global Health 2020; 16:5.

12. Friedman EA, Gostin LO, Kavanagh MM, Periago MR, Marmot M, Coates A, Binagwaho A, Mukherjee J, Chowdhury M, Robinson T, Veloso VG, Wang C, Were M. Putting health equity at heart of universal coverage-the need for national programmes of action. BMJ 2019; 367:15901. 
13. Labonté R. Health Promotion in an Age of Normative Equity and Rampant Inequality. Int $J$ Health Policy Manag 2016; 5(12):675-682.

14. Silva DAJ, Tavares MFL. Ação intersetorial: potencialidades e dificuldades do trabalho em equipes da Estratégia Saúde da Família na cidade do Rio de Janeiro. Saúde Debate 2016; 40(111):193-205.

15. Shankardass K, Solar O, Murphy K, Freiler A, Bobbili S, Bayoumi A, O'Campo P. Health in all policies: a snapshot for Ontario, results of a realist-informed scoping review of the literature. Toronto: St Michael's; 2011.

16. Solar O, Valentine N, Rice M, Albrecht D. Moving forward to equity in health: what kind of intersectoral action is needed? An approach to an intersectoral typology. Prepared for the 7th Global Conference on Health Promotion, "Closing the Implementation Gap", 26-30 October 2009, Nairobi, Kenya.

17. Stewart MJ, Neufeld A, Harrison MJ, Spitzer D, Hughes K, Makwarimba E. Immigrant women family caregivers in Canada: implications for policies and programmes in health and social sectors. Health Soc Care Community 2006; 14(4):329-340.

18. Perera MALR. Intersectoral action for health in Sri Lanka. Sri Lanka: Institute for Health Policy; 2006.

19. Zunzunegui MV, Béland F. Políticas intersectoriales para abordar el reto del envejecimiento activo. Informe SESPAS 2010. Gac Sanit 2010; 24(1):68-73.

20. Rantala R, Bortz M, Armada F. Intersectoral action: local governments promoting health. Health Promot Int 2014; 29(Suppl. 1):82-102.

21. Buse C. Intersectoral action for health equity as it relates to climate change in Canada: contributions from critical systems heuristics. J Eval Clin Pract 2013; 19(6):1095-1100.

22. Azevedo E, Pelicioni MCF, Westpal MF. Práticas intersetoriais nas políticas públicas de promoção de saúde. Physis 2012: 22(4):1333-1356.

23. Anaf J, Baum F, Freeman T, Labonte R, Javanparast $S$, Jolley G, Lawless A, Bentley M. Factors shaping intersectoral action in primary health care services. Aust $N$ Z J Public Health 2014; 38(6):553-559.

24. Tubbing L, Harting J, Stronks K. Unravelling the concept of integrated public health policy: Concept mapping with Dutch experts from science, policy, and practice. Health Policy 2015; 119(6):749-759.

25. Weiss D, Lillefjell M, Magnus E. Facilitators for the development and implementation of health promoting policy and programs - a scoping review at the local community level. BMC Public Health 2016; 16:140.

26. Clavier C, Gagnon F. L'action intersectorielle en santé publique ou lorsque les institutions, les intérêts et les idées entrent en jeu. The Innovation Journal: The Public Sector innovation Journal 2013; 18(2):2.

27. Wimmer GF, Figueiredo GO. Ação coletiva para qualidade de vida: autonomia, transdisciplinaridade e intersetorialidade. Cien Saude Colet 2006: 11(1):145154.

28. Dias MSA, Parente JRF, Vasconcelos MIO, Dias FAC. Intersetorialidade e Estratégia Saúde da Família: tudo ou quase nada a ver? Ciencia Saude Colet 2014; 19(11):4371-4382.

29. Mannheime et al. Introducing Health Impact Assessment: an analysis of political and administrative intersectoral working methods. Eur J Public Health 2007; 17(5):526-531.
30. Kalegaonkar A, Brown D. Intersectoral cooperation: lessons for practice. Boston: Institute for Development Research; 2000.

31. Axelsson R, Axelsson S. Integration and collaboration in public health - a conceptual framework. Int $J$ Health Plann Manage 2006; 21:75-88.

32. Shankardass K, Solar O, Murphy K, Greaves L, O'Campo P. A scoping review of intersectoral action for health equity involving governments. Int J Public Health, 2012: 57(1):25-33.

33. Rissel C, Rowling L. Intersectoral collaboration for the development of a national framework for health promoting schools in Australia. J Sch Health 2000; 70(6):248-250.

34. El Ansari. Collaborative research partnerships with disadvantaged communities: challenges and potential solutions. Public Health 2005; 119(9):758-770.

35. Lal S, Mercier C. Intersectoral action to employ individuals with mental illness: lessons learned from a local development initiative. Work 2009; 33(4):427-437.

36. Rantala R, Bortz M, Armada F. Intersectoral action: local governments promoting health. Health Promot Int 2014;29(1):192-102.

37. Keon, Pépin. Fourth report of the subcommittee on population health of the standing senate committee on social affairs, science and technology. Science and Technology, Parliament of Canada; 2008.

38. Repetto F. Coordinación de políticas sociales: abordaje conceptual y revisión de experiencias latinoamericanas. In: Acuña $\mathrm{CH}$, Nogueira RM, Repetto F. Proyecto de Modernización del Estado. Los desafíos de la coordinación y la integralidad de las políticas y gestión pública en América Latina. Buenos Aires: Jefatura de Gabinete de Ministros; 2009. p. 47-84.

39. Cabrero EM, Zabaleta DS. ¿Cómo construir una mística intergubernamental en la política social? Análisis de cuatro experiencias latino-americanas. Revista del CLAD Reforma y Democracia 2009; 43.

40. Molina G, Licha I. Coordinación de La Política: criterios para avanzar. Washington: INDES/BID; 2005.

41. Horwath J, Morrison T. Collaboration, integration and change in children's services: critical issues and key ingredients. Child Abuse Negl 2007; 31(1):55-69.

42. Winkworth G, White M. Australia's Children 'Safe and Well'? Collaborating with Purpose Across Commonwealth Family Relationship and State Child Protection Systems. Australian Journal of Public Administration 2011; 70(1):1-14.

43. Serrate PCF. A intersetorialidade: conceptualização: panorama internacional e cubana. Havana: Escola Nacional de Saúde Pública; 2013.

44. Mannheimer LN, Gulis G, Lehto J, Ostlin P. Introducing Health Impact Assessment: an analysis of political and administrative intersectoral working methods. Eur J Public Health 2007; 17(5):526-531.

45. Solar O, Irwin A. A conceptual framework for action on the social determinants of health, in Social Determinants of Health Discussion Paper 2. (Policy and Practice). Geneva: WHO; 2010.

Artigo apresentado em 23/09/2020

Aprovado em 17/02/2021

Versão final apresentada em 19/02/2021

Editores-chefes: Romeu Gomes, Antônio Augusto Moura da Silva 